\title{
TITANIA SILICATE / FUSED QUARTZ GLASSBLOWING FOR 3-D FABRICATION OF LOW INTERNAL LOSS WINEGLASS MICRO-STRUCTURES
}

\author{
D. Senkal, C.R. Raum, A.A. Trusov, and A.M. Shkel \\ MicroSystems Laboratory, University of California, Irvine, CA, USA
}

\begin{abstract}
We report, for the first time, a fabrication process for building highly smooth, symmetric 3-D dynamic wineglass structures using low internal loss materials, namely fused quartz and ultra low expansion titania silicate glass (ULE TSG). The approach consists of a high temperature (at $1650{ }^{\circ} \mathrm{C}$ ) micro-glassblowing process and a novel inverted-wineglass architecture that provides self-aligned stem structures. An in-house process capability of $1800{ }^{\circ} \mathrm{C}$ glassblowing with a rapid cooling rate of $500{ }^{\circ} \mathrm{C} / \mathrm{min}$ was developed. Feasibility of the process has been demonstrated by fabrication of TSG wineglass micro-structures. The method may enable new classes of TSG and fused quartz MEMS devices with extremely low surface roughness, $0.23 \mathrm{~nm}$ surface average $(\mathrm{Sa})$, intrinsically low internal losses $\left(\mathrm{Q}_{\mathrm{TED}}>5 \mathrm{E}+10\right)$ and dynamically balanced structures.
\end{abstract}

\section{INTRODUCTION}

Maximization of the quality (Q) factor is key to enhancing performance of vibratory MEMS devices in demanding signal processing, timing and inertial applications [1]. The macro-scale hemispherical resonator gyroscope (HRG) with Q-factors over $25 \mathrm{E}+6$ [2] motivates the investigation of 3-D fused quartz microwineglass structures for use as vibratory elements.

With the emergence of novel fabrication techniques batch fabrication of 3-D wineglass structures are becoming possible. For instance, hemispherical shells fabricated by deposition of polysilicon [3] or silicon nitride [4] thin films into isotropically etched cavities have recently been demonstrated. Alternative fabrication techniques include "3-D SOULE" process for fabrication of mushroom and concave shaped spherical structures [5] as well as blow molding of bulk metallic glasses into preetched cavities [6]. However, MEMS wineglass resonators with sufficient smoothness and low thermoelastic dissipation (TED) have not yet been demonstrated in the literature. To take full advantage of the 3-D wineglass architecture, fabrication techniques with low surface roughness as well as materials with high isotropy and low TED are desired.

It has been demonstrated that MEMS devices can reach the fundamental $\mathrm{Q}_{\mathrm{TED}}$ limit by using a combination of balanced mechanical design and vacuum packaging with getters [7]. TED is caused by local temperature fluctuations due to vibration and the associated irreversible heat flow, which results in entropic dissipation. TED can be reduced either by decoupling the mechanical vibrations from the thermal fluctuations or by using materials with low coefficient of thermal expansion (CTE). This paper focuses on materials with low CTE, such as fused quartz $\left(0.5 \mathrm{ppm} /{ }^{\circ} \mathrm{C}\right)$ or ultra low expansion titania silicate glass $\left(0.03 \mathrm{ppm} /{ }^{\circ} \mathrm{C}\right)$, which can provide a dramatic increase in fundamental $\mathrm{Q}_{\mathrm{TED}}$ limit $\left(\mathrm{Q}_{\mathrm{TED}}>7 \mathrm{E}+10\right.$ for a $\mathrm{TSG}$ wineglass). However, when compared to silicon, titania silicate glass and fused quartz dry etching suffers from order of magnitude higher surface roughness, lower mask selectivity and aspect ratios [8].

Pyrex glassblowing at $850^{\circ} \mathrm{C}$ on a silicon substrate was previously demonstrated for fabrication of smooth, symmetric 3-D structures [9-11]. However, TSG glassblowing requires upwards of $1600{ }^{\circ} \mathrm{C}$ glassblowing temperature due to its higher softening point, which prevents the use of fabrication processes that rely on a

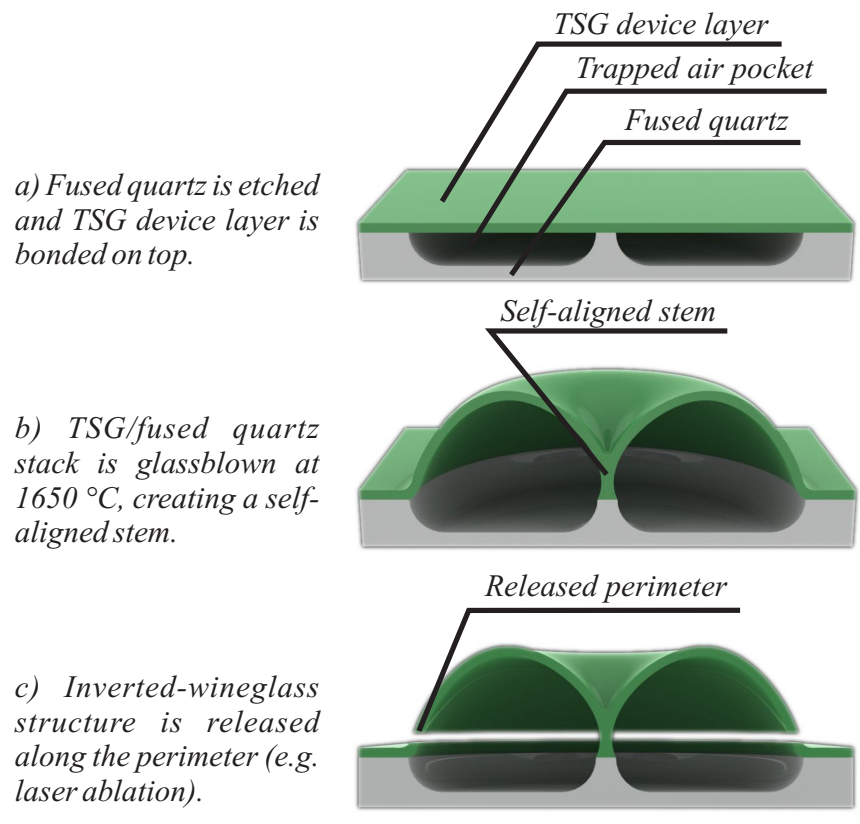

Figure 1: High temperature glassblowing process for fabrication of ULE TSG / fused quartz inverted-wineglass structures.

silicon substrate. This paper explores the hypothesis that high temperature glassblowing $\left(1650^{\circ} \mathrm{C}\right)$, may serve as an enabling mechanism for wafer-scale fabrication of TSG / fused quartz 3-D wineglass structures. The approach consists of a high temperature micro-glassblowing process and a novel inverted-wineglass architecture that provides self-aligned stem structures, Fig. 1. An in-house process capability of $1800{ }^{\circ} \mathrm{C}$ glassblowing with a rapid cooling rate of $500{ }^{\circ} \mathrm{C} / \mathrm{min}$ was developed. Feasibility of the process has been demonstrated by fabrication of TSG / fused quartz micro-wineglass structures.

\section{DESIGN TRADE-OFFS}

To analyze the effects of geometric design parameters as well as the material choice on fundamental Q-factor limits, parametric finite element analysis (FEA) was conducted using Comsol Multiphysics Package.

\section{Anchor Loss}

The focus of the analysis was to compare the invertedwineglass architecture proposed in this paper vs. previously fabricated glassblown structures with large attachment diameter, Fig. 2 [11]. The structure in Fig. 2(a) was fabricated by first glassblowing a spherical structure through a stencil layer and then laser micromachining the cap to create a wineglass structure.

The structure in Fig. 2(a) had a shell diameter of $1142 \mu \mathrm{m}$, anchor diameter of $600 \mu \mathrm{m}$ and average thickness of $4 \mu \mathrm{m}$, which gives roughly 1:2 attachment to shell diameter ratio. In contrast, the inverted-wineglass structure with the integrated stem, Fig. 2(b), had a shell diameter of $4200 \mu \mathrm{m}, 300 \mu \mathrm{m}$ anchor diameter and an average thickness of $80 \mu \mathrm{m}$, giving a 1:14 anchor to shell diameter ratio. 


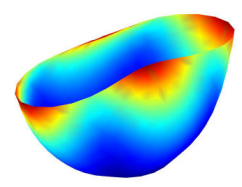

(a) without stem

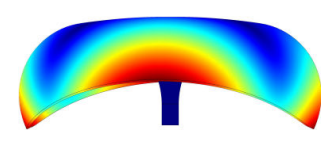

(b) with stem

\begin{tabular}{lccccc}
\hline & \multicolumn{4}{c}{$\mathbf{Q}_{\text {TED }}$} & Q $_{\text {anchor }}$ \\
\cline { 2 - 5 } & Silicon & Pyrex & FQ & TSG & \\
\hline (a) w/o stem & $1 \mathrm{E}+05$ & $1 \mathrm{E}+06$ & $4 \mathrm{E}+07$ & $1 \mathrm{E}+10$ & $3 \mathrm{E}+03$ \\
(b) with stem & $6 \mathrm{E}+04$ & $7 \mathrm{E}+06$ & $2 \mathrm{E}+07$ & $\mathbf{7 E}+\mathbf{1 0}$ & $\mathbf{5 E}+\mathbf{1 0}$ \\
\hline
\end{tabular}

Figure 2: Fundamental $Q_{T E D}$ and $Q_{\text {anchor limits for (a) without }}$ stem, (b) with stem, obtained using finite element analysis.

To simulate the accoustic loss in an infinite medium, a perfectly matched layer (PML) was used for the substrate domain. PML works by absorbing accoustic waves over a large frequency range at any non-zero angle of incidence. The simulation was run for perfectly symmetric structures, neglecting the contribution of mass imbalance to the anchor loss. For this reason, the values obtained from FEA represent the fundamental anchor loss limit of the structures. The wineglass structure with 1:2 anchor to shell diameter ratio Fig. 2(a) had a fundamental $Q_{\text {anchor }}$ limit of 3000, which is in close agreement with the experimentally obtained quality factor of 1256 [11]. In contrast, the analysis of the wineglass structure with the integrated stem (1:14 ratio), Fig. 2(b), showed virtually zero anchor loss $\left(\mathrm{Q}_{\text {anchor }}>5 \mathrm{E}+10\right)$.

\section{Thermoelastic Dissipation}

The goal of the analysis was to understand the effect of CTE on $\mathrm{Q}_{\mathrm{TED}}$. Four different materials: Silicon, pyrex, fused quartz and TSG were investigated, Fig. 2. Energy loss caused by TED was analyzed using a coupled thermo-mechanical model. The model was solved for the $n=2$ wineglass modes, and $Q_{T E D}$ values were extracted from the ratio of the real and imaginary parts of the eigenfrequencies. The difference in $\mathrm{Q}_{\mathrm{TED}}$ between the two geometries was limited to within one order of magnitude, whereas the material choice had a huge impact on the $\mathrm{Q}_{\mathrm{TED}}$. TSG (with the lowest CTE among the materials investigated) had the highest fundamental $\mathrm{Q}_{\mathrm{TED}}$ value at $7 \mathrm{E}+10$, which was followed by fused quartz at $\mathrm{Q}_{\mathrm{TED}}>2 \mathrm{E}+7$.

\section{FABRICATION PROCESS}

Fabrication process for TSG wineglass structures consists of four main steps, namely: (1) Etching of the fused quartz (or fused silica) substrate, (2) bonding of TSG device layer to fused quartz, (3) glassblowing, and finally (4) releasing the wineglass structure by etching around the perimeter, Fig. 1. The process starts by LPCVD deposition of a $2 \mu \mathrm{m}$ PolySi hard mask onto the fused quartz substrate. After which, the cavity openings were defined on the PolySi hard-mask using RIE. Then, $\sim 150 \mu \mathrm{m}$ deep toroidal cavities were wet etched into the substrate wafer using concentrated HF (49\%). In order to establish the etch rate of HF on fused quartz and TSG, 75 minute test runs were performed at room temperature. Etch depth was measured every 15 minutes by stopping the etch and measuring using a DEKTAK 3 profilometer. Linear regression fits showed an etch rate of $1.07 \mu \mathrm{m} / \mathrm{min}$ for fused quartz and $2.86 \mu \mathrm{m} / \mathrm{min}$ for TSG, with a linearity of $\mathrm{R}^{2}=0.996$ and $\mathrm{R}^{2}=0.997$ respectively. Once the etch was complete, PolySi layer was stripped and the wafers were thoroughly cleaned using RCA clean.

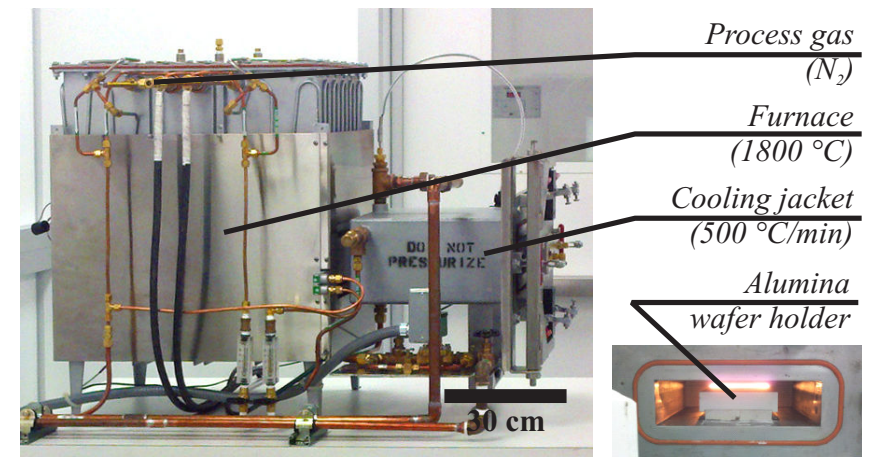

Figure 3: Process capability of $1800^{\circ} \mathrm{C}$ glassblowing with a rapid cooling rate of $500^{\circ} \mathrm{C} / \mathrm{min}$ was developed.

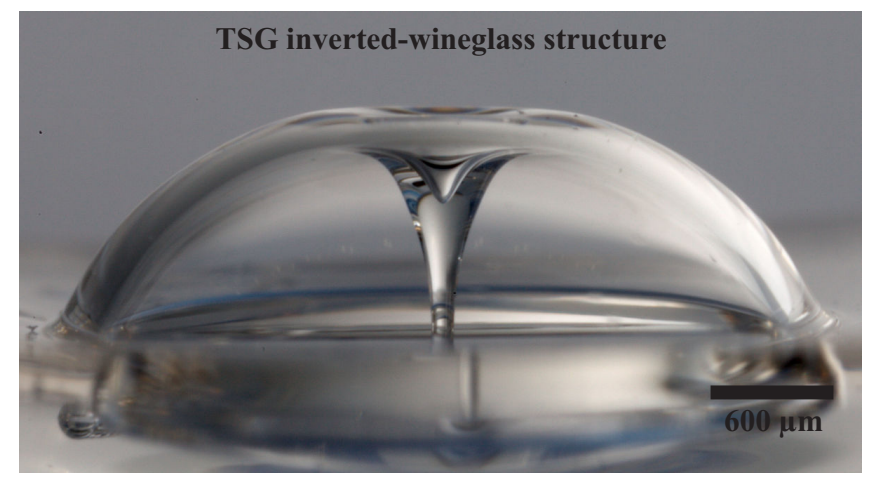

Figure 4: Optical photograph of glassblown TSG invertedwineglass structure. Outer diameter is $4200 \mu \mathrm{m}$.

The next step of the fabrication process is bonding of the TSG device layer onto the etched fused quartz wafer. Due to the subsequent high temperature glassblowing process the bond needs to survive up to $1650{ }^{\circ} \mathrm{C}$, which prevents the use of intermediate materials. For this reason, a plasma activated fusion bonding process was developed, Fig. 1(a). The bond is performed by plasma activating the TSG and fused quartz wafers and then bringing them into optical contact. Since the process relies on hydrogen bonds to keep the two wafers together, highly polished and clean surfaces $(<1 \mathrm{~nm} \mathrm{Sa})$ are required. Once cured, the bond creates a seamless hermetic seal around the etched cavities without using any intermediate material.

The TSG / fused quartz wafer stack is then glassblown at $1650{ }^{\circ} \mathrm{C}$ in a custom-built high temperature furnace with a rapid cooling rate of $500{ }^{\circ} \mathrm{C} / \mathrm{min}$, Fig. 3. The furnace consists of two main chambers that are connected to each other through a third vestibule chamber. The first chamber is used for heating and can go up to $1800{ }^{\circ} \mathrm{C}$, the second chamber is enveloped by a water cooled jacket, that maintains $<200{ }^{\circ} \mathrm{C}$ temperature. The samples are transported between the heating and cooling chambers by using a sliding alumina wafer holder. A typical glassblowing run involves keeping the wafer stack at glassblowing temperature for one minute and then extracting the wafer stack into the water cooled jacket for solidification.

During glassblowing, two phenomenon occur simultaneously: TSG layer becomes viscous due to the elevated temperature, the air inside the etched cavity expands, creating the 3-D glassblown structure. Because the TSG wafer is bonded both around the circular cavity and the cylindrical post in the middle, the glassblown structure creates a self-aligned TSG stem, Fig. 4. 


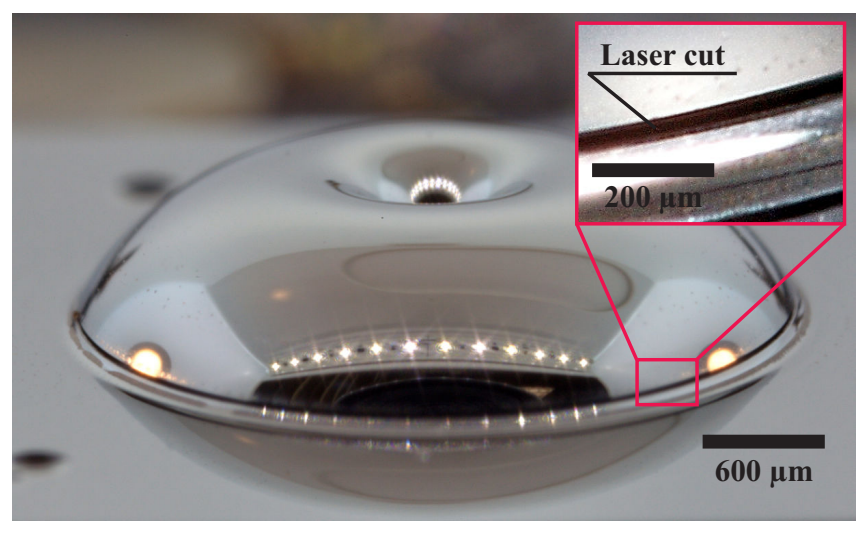

Figure 5: Optical photograph of inverted-wineglass, released along the perimeter using laser ablation and coated with iridium.

The final step of the fabrication process is to release the wineglass around its perimeter, which can be accomplished by laser ablation or dry etching of the device layer. The wineglass structure in Fig. 5 was released with laser ablation, using a 2-axis laser micromachining system, Resonetics RapidX 250. The system was upgraded to 3-axis by installing a custom built rotary stage assembly from National Aperture, Inc. The wineglass structure was mounted onto the rotary stage and its axis of symmetry was aligned with the rotation axis with the help of an $x-y$ stage. Laser ablation was performed by focusing the laser onto the perimeter of the wineglass at a perpendicular angle and rotating the wineglass structure at constant angular velocity. For the laser source, an ArF (193 nm) excimer laser (Coherent COMPexPRO 110) was used with $20 \mathrm{~ns}$ pulse duration, $50 \mathrm{~Hz}$ repetition rate and a laser spot size of $40 \mu \mathrm{m}$.

\section{METROLOGY}

In this section we analyze the surface roughness and the material composition of TSG device layer before and after glassblowing.

\section{Surface Roughness}

Surface roughness measurements of TSG glassblown samples were performed using an atomic force microscope (AFM) from Pacific Nanotechnology (Nano-R). With a sensor noise level of $<0.13 \mathrm{~nm}$ in the z-direction, Nano-R can resolve sub-nanometer features. Samples were cleaned using standard solvent clean (Acetone, IPA, methanol) before each scan. No additional treatment was performed on the samples. The AFM was run in tapping mode, using a $10 \mathrm{~nm}$ radius probe tip (Agilent U3120A).

Surface roughness of the samples before and after glassblowing were analyzed, with the hypothesis that glassblowing can improve the surface roughness. Highly polished TSG wafers were used for the device layer, which was verified by AFM scans, showing a surface roughness of $0.40 \mathrm{~nm} \mathrm{Sa,} \mathrm{Fig.} \mathrm{6(b).}$ Characterization of the glassblown samples showed a two-fold improvement in surface roughness, down to $0.23 \mathrm{~nm} \mathrm{Sa}$, Fig 6(a). We also observed that the angstrom level scratches in Fig 6(b), associated with the lapping operation, disappeared after glassblowing, Fig 6(a), confirming the hypothesis.

Two-fold improvement in surface roughness is attributed to viscous flow of the glass layer and the associated surface tension forces. As the glassblowing is performed above the glass softening temperature, TSG device layer becomes viscous and the surface tension forces become active, working towards minimizing the

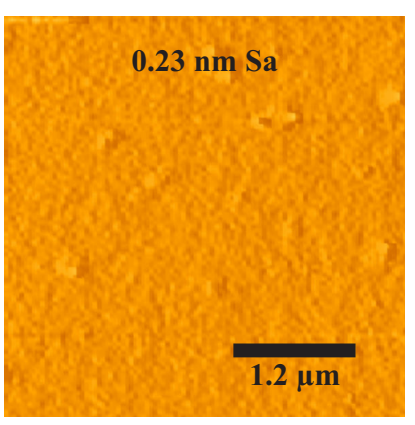

(a) After glassblowing

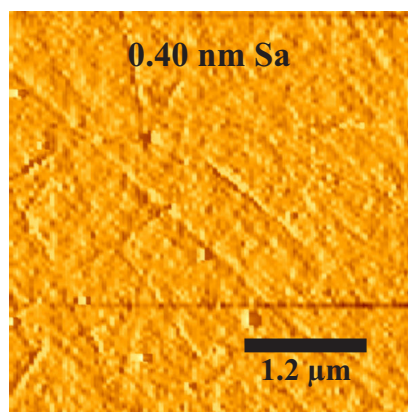

(b) Before glassblowing

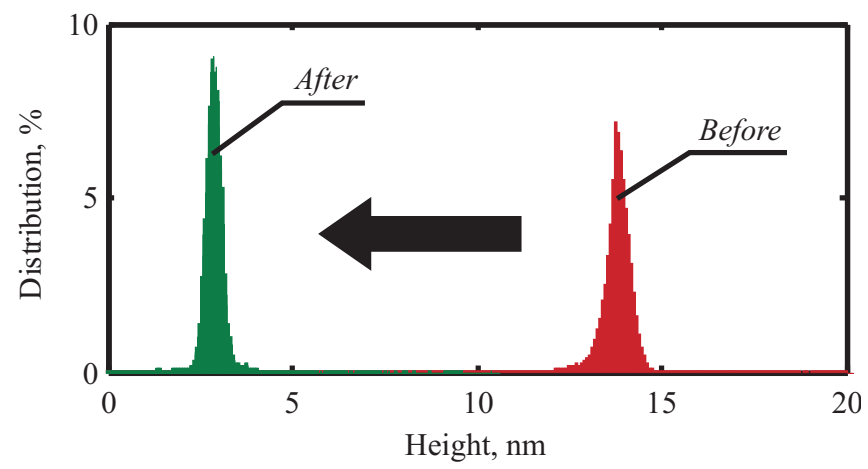

Figure 6: AFM surface profiles of TSG, (a) before and (b) after glassblowing. Glassblowing creates extremely smooth $(0.2 \mathrm{ppm}$ relative roughness) TSG structures.

surface area of the glass structure. This creates an effect analogous to "stretching out" the wrinkles on the surface, lowering the surface roughness.

\section{Material Composition}

For resonant and optical applications, it is critical that TSG retains its original material composition and properties after glassblowing, which are structural integrity, material uniformity and optical transparency. We found that glassblowing temperature and the rate of cooling are the most important parameters that affect the quality of the TSG layer after glassblowing.

The structure in Fig. 7 was glassblown using a conventional high temperature furnace at $1600^{\circ} \mathrm{C}$, which does not allow removal of the samples at elevated temperatures. For this reason, the structure was left to cool-down to room temperature over an 8 hour period. Recrystallization as well as micro-cracks were observed on the surface. In order to establish the nature of the recrystallization electron dispersive spectroscopy (EDS) was employed. Philips XL-30 FEG SEM with a Thermo Scientific UltraDry silicon drift X-ray detector was used for EDS characterization. An acceleration voltage of $10 \mathrm{kV}$ was used at $10 \mathrm{~mm}$ working distance, and samples were coated with $5 \mathrm{~nm}$ of sputtered iridium to prevent charging.

EDS analysis of the crystals in Fig. 7 revealed higher concentrations of titanium, implying that $\mathrm{TiO}_{2}$ is exsolving from the $\mathrm{SiO}_{2} / \mathrm{TiO}_{2}$ matrix. In contrast, the structure in Fig. 8 was glassblown using rapid cooling by bringing the temperature of the sample from $1650{ }^{\circ} \mathrm{C}$ to $\sim 200{ }^{\circ} \mathrm{C}$ within a minute. No microcracks or recrystallization were observed, as can be validated by the optical transparency. EDS spectral plots showed homogeneous $\mathrm{SiO}_{2}$ and $\mathrm{TiO}_{2}$ distribution in Fig. 8 as opposed to heterogeneous distribution in Fig. 7. 

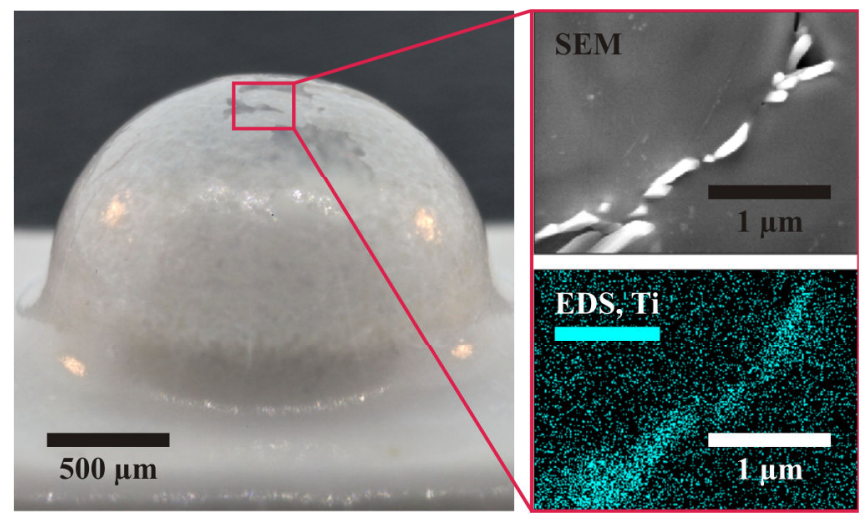

Figure 7: Slow cooling of TSG (> $8 \mathrm{hr}$.) causes recrystallization.
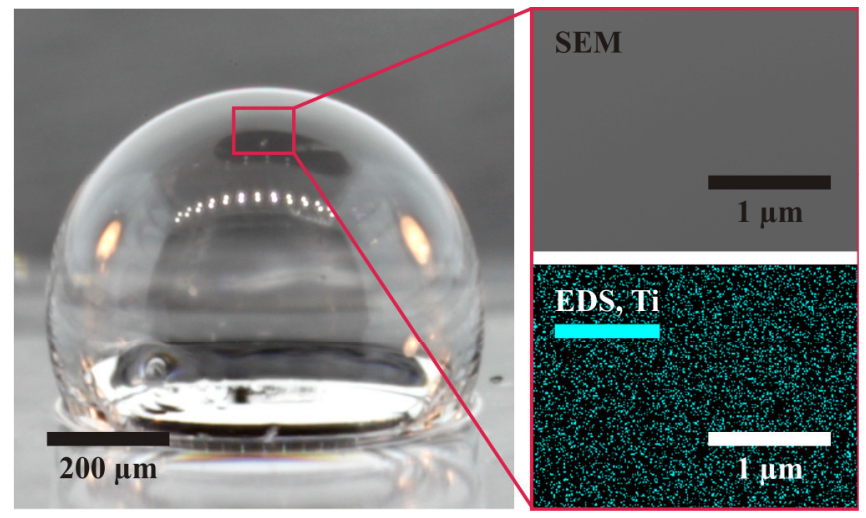

Figure 8: Glassblowing with rapid cooling of TSG $(<1$ min.) prevents recrystallization.

EDS was used to obtain the spectral signatures of TSG before and after glassblowing. No change in the composition of TSG was observed after glassblowing, Fig. 9. EDS spectrums also revealed 7 to 8 weight percent of $\mathrm{TiO}_{2}$ in TSG, which is in agreement with the nominal $\mathrm{TiO}_{2}$ concentration of Corning ULE TSG.

\section{CONCLUSIONS}

A new high temperature micro-glassblowing process for fabrication of 3-D low internal loss ULE TSG wineglass structures was developed and experimentally demonstrated. The process was enabled by the development of an in-house high temperature glassblowing capability of $1800^{\circ} \mathrm{C}$ with a cooling rate of $>500{ }^{\circ} \mathrm{C} / \mathrm{min}$ and plasma activated fusion bonding of TSG to fused quartz. EDS spectral analysis of TSG before and after glassblowing revealed that the material retained its properties after glassblowing with no recrystallization or change in glass composition. AFM surface scans of the glassblown structures showed extremely low surface roughness $(0.23 \mathrm{~nm} \mathrm{Sa})$. A novel inverted-wineglass architecture was also implemented, providing self-aligned stem structures with a fundamental anchor loss limit of $\mathrm{Q}_{\text {anchor }}>7 \mathrm{E}+10$. The method may enable new classes of TSG / fused quartz MEMS devices with extremely low surface roughness, intrinsically low internal losses $\left(\mathrm{Q}_{\mathrm{TED}}>5 \mathrm{E}+10\right)$ and dynamically balanced structures.

\section{ACKNOWLEDGEMENTS}

This material is based upon work supported by DARPA/SPAWAR under Grant N66001-10-1-4074 (Program Manager Tayo Akinwande) \& DARPA grant W31P4Q-11-1-0006.

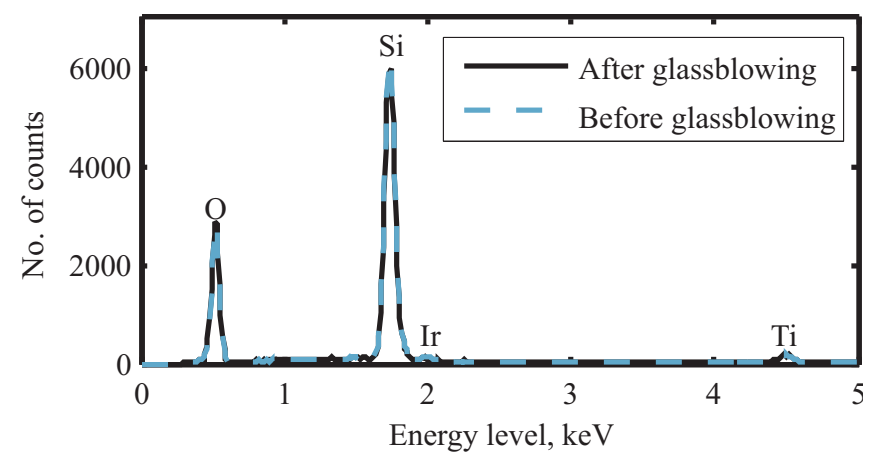

Figure 9: EDS spectral analysis of TSG reveals that composition of TSG does not change after glassblowing.

\section{REFERENCES}

[1] M. Weinberg, R. Candler, S. Chandorkar, J. Varsanik, T. Kenny, A. Duwel, "Energy Loss in MEMS Resonators and the Impact on Inertial and RF Devices," Proc. Transducers'09, Denver, Colorado, USA, June 21-25, 2009, pp. 688-695.

[2] D.M. Rozelle, "The hemispherical resonator gyro: From wineglass to the planets", in Proc. AAS/AIAA Space Flight Mechanics Meeting, Feb. 2009, pp. 1157-1178.

[3] L. D. Sorenson, X. Gao, F. Ayazi, "3-D Micromachined Hemispherical Shell Resonators With Integrated Capacitive Transducers," Proc. IEEE MEMS'12, Paris, France, Jan. 29 Feb. 2, 2012, pp. 168-171.

[4] C.L. Fegely, D.N. Hutchison, S.A. Bhave, "Isotropic etching of 111 SCS for wafer-scale manufacturing of perfectly hemispherical silicon molds," Proc. Transducers'11, Beijing, China, June 5-9, 2011, pp 2595-2598.

[5] K. Visvanathan, Li Tao, Y. B. Gianchandani, "3-D-soule: A fabrication process for large scale integration and micromachining of spherical structures", Proc. IEEE MEMS'11, Jan. 21 - 27, 2011, pp. 45-48.

[6] B. Sarac, G. Kumar, T. Hodges, S. Ding, A. Desai, J. Schroers, "Three-Dimensional Shell Fabrication Using Blow Molding of Bulk Metallic Glass," IEEE J. Microelectromech. Syst., vol. 20, no. 1, pp. 28-36, 2011.

[7] I.P. Prikhodko, S.A. Zotov, A.A. Trusov, A.M. Shkel, "Subdegree-per-hour silicon MEMS rate sensor with 1 million Qfactor," Proc. Transducers'11, Beijing, China, June 5-9, 2011, pp. 2809-2812.

[8] Chen, W., Sugita, K., Morikawa, Y., Yasunami, S., Hayashi, $\mathrm{T}$., \& Uchida, $\mathrm{T}$, "Application of magnetic neutral loop discharge plasma in deep silica etching," J. Vacuum Science \& Technology, vol. 19, no.6, pp. 2936-2940, 2001.

[9] E.J. Eklund, A.M. Shkel, "Glass Blowing on a Wafer Level," IEEE J. Microelectromech. Syst., 16(2), pp. 232-239, 2007.

[10] I.P Prikhodko, S.A. Zotov, A.A. Trusov A.M. Shkel, "Microscale Glass-Blown Three-Dimensional Spherical Shell Resonators," IEEE J. Microelectromech. Syst., vol. 20, no. 3, pp.691-701, 2011.

[11] D. Senkal, I. P. Prikhodko, A. A. Trusov, A. M. Shkel, "Micromachined 3-D Glass-Blown Wineglass Structures for Vibratory MEMS Applications," Technologies or Future Micro-Nano Manufacturing, Napa, California, USA, August 8 - 10, 2011, pp. 166-169.

\section{CONTACT}

* A.A. Trusov, tel: +1-949-824-6314; atrusov@uci.edu. 